\title{
Image-guided video-assisted thoracoscopic surgery for small ground glass opacities: a case series
}

\author{
Hsin-Yueh Fang, Yin-Kai Chao, Ming-Ju Hsieh, Chih-Tsung Wen, Pei-Hsuan Ho, Wei-Jiun Tang, \\ Yun-Hen Liu \\ Division of Thoracic Surgery, Chang Gung Memorial Hospital, College of Medicine, Chang Gung University, Taoyuan, Taiwan \\ Contributions: (I) Conception and design: HY Fang, MJ Hsieh; (II) Administrative support: YH Liu, CT Wen; (III) Provision of study materials or \\ patients: MJ Hsieh, YK Chao; (IV) Collection and assembly of data: HY Fang, PH Ho, WJ Tang, YK Chao; (V) Data analysis and interpretation: HY \\ Fang, YK Chao; (VI) Manuscript writing: All authors; (VII) Final approval of manuscript: All authors. \\ Correspondence to: Yun-Hen Liu, MD. Division of Thoracic Surgery, Chang Gung Memorial Hospital-Linko, Chang Gung University, Taoyuan, \\ Taiwan. Email: 15710111@cgmh.org.tw.
}

\begin{abstract}
Background: This case series demonstrated the feasibility of the image-guided video-assisted thoracoscopic surgery (iVATS) for localization and removal of ground glass opacities (GGOs). The procedure was performed in a hybrid operating room (OR) using C-arm cone-beam computed tomography (CBCT) equipped with a laser-guided navigation system.
\end{abstract}

Methods: Between October $1^{\text {st }} 2016$ to July $31^{\text {st }} 2017,14$ consecutive patients presenting with GGOs underwent iVATS procedure. The efficacy and safety of the procedure were assessed through a retrospective chart review.

Results: The median GGOs size was $7 \mathrm{~mm}$ [interquartile range (IQR): 4-10 mm] with a median depthto-size (D-S) ratio of 1.16 (IQR: 0-2.3). All of the lesions were visible on intraoperative CBCT images and localizations were successful in all patients with a median localization time of $22 \mathrm{~min}$ (IQR: 16-44 min). No patient required a conversion to thoracotomy. There was no operative mortality and the median length of postoperative stay was 4 days (IQR: 3-6 days). The final pathological diagnoses were as follows: primary lung cancer $(\mathrm{n}=6)$, lung metastases $(\mathrm{n}=2)$, and benign lung lesions $(\mathrm{n}=6)$.

Conclusions: Our study suggests the iVATS could be a helpful tool for single-stage detection and removal of GGOs.

Keywords: Computed tomography (CT); ground glass opacities (GGOs); video-assisted thoracoscopic surgery (VATS); ARTIS zeego

Received: 28 August 2017; Accepted: 15 September 2017; Published: 18 October 2017.

doi: 10.21037 /jovs.2017.09.08

View this article at: http://dx.doi.org/10.21037/jovs.2017.09.08

\section{Introduction}

Lung cancer is the leading cause of cancer death worldwide. Due to the positive result of lung cancer screening trial, using low-dose computed tomography (CT) for early lung cancer detection has gained popularity $(1,2)$. As a consequence, thoracic surgeons are often being challenged with the removal of undiagnosed ground glass opacities (GGOs) with high probability of malignancy. However, GGOs are generally not visible to videoscopic observation and unlikely to be palpable through thoracoscopic instrument. A high conversion rate from video-assisted thoracoscopic surgery (VATS) to thoracotomy has been reported when lung nodules are less than $10 \mathrm{~mm}$ in diameter or located more than $5 \mathrm{~mm}$ below the pleural surface (3).

In order to minimize the likelihood of unplanned conversion to thoracotomy, a two-stage steps approach which consisted with preoperative tumor localization in an interventional CT suite followed by patient transfer to an operating room (OR) has been utilized. However, this 


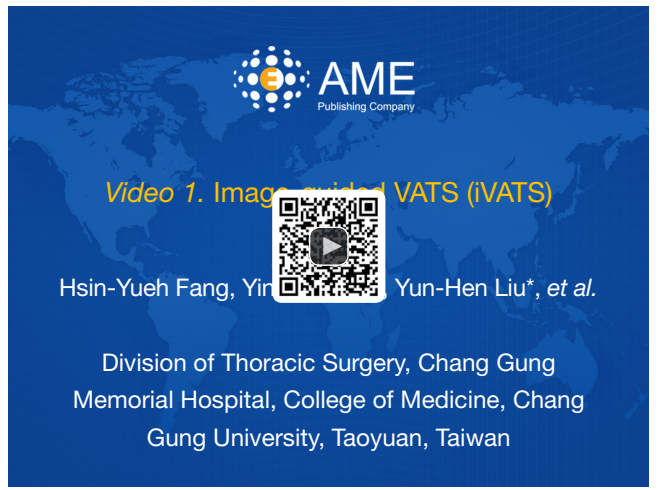

Figure 1 Image-guided VATS (iVATS) workflow (13). (I) Preoperative planning on traditional computed tomography; (II) iVATS procedure was performed in a hybrid OR, with a $\mathrm{C}$-arm CBCT and a Magnus surgical table; (III) After anesthesia, the patient was placed in the planned position; (IV) Pre-procedural CBCT scan for needle path planning; (V) Needle entry guided by laser-target cross; (VI) Post-procedural CBCT scan for needle path confirmed; (VII) Tumor resection with hook wire guided. Available online: http://www.asvide.com/articles/1752

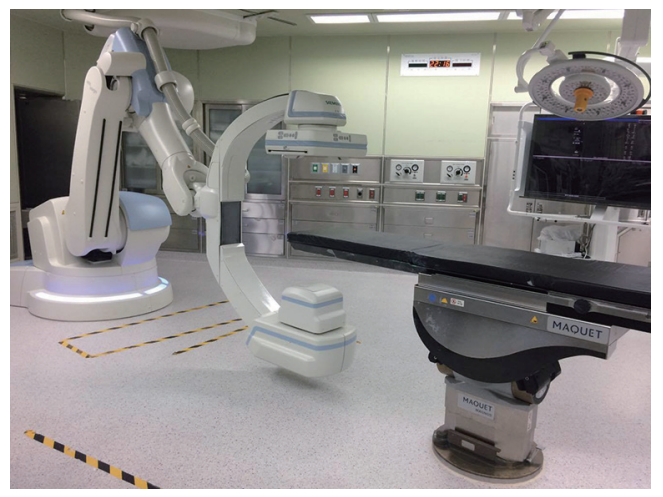

Figure 2 The arrangement of a hybrid OR, which included a C-arm cone-beam computed tomography (CBCT) and a Magnus surgical table.

workflow requires a smooth coordination between the CT suite and the OR. Otherwise, risk of complications (including pneumothorax, hemothorax, wire dislodgement, and dye fading) increased substantially when the waiting time increased (4-7).

In recent years, single-stage image-guided VATS (iVATS) has been proposed to overcome the known drawbacks of two-stage approach. Through a hybrid OR design, patients could undergo both localization and operation in the same environment. Although there are several studies showing the feasibility of iVATS, most of the studies were focused on solid lesions (8-10). The feasibility to localize GGO lesion through a cone-beam CT (CBCT) has never been reported. To this aim, we report our initial experience concerning the use of iVATS for simultaneous localization and removal of GGOs.

\section{Methods}

\section{Study patients}

Consecutive patient with undiagnosed GGOs and received iVATS between October $1^{\text {st }} 2016$ and July $31^{\text {st }}$, 2017 were eligible. The study protocol was approved by the Institutional Review Board of the Chang Gung Memorial Hospital (CGMH-IRB 201600671A3). Written informed consent was obtained from all participants. The GGO is defined as an obscure opacity with bronchial and vascular seen in CT image (11).

\section{iVATS workflow}

The procedural workflow has been previously described in detail (12) and shown on the video (Figure 1). The entire iVATS procedure was performed in a hybrid OR (Figure 2) in which a C-arm CBCT (ARTIS zeego; Siemens Healthcare GmbH, Erlangen, Germany) and a Magnus surgical table (Maquet Medical Systems, Wayne, NJ, USA) were available. A single team of thoracic surgeons performed both localization and surgery. After general anesthesia completed, the patient was placed in the planned position. All pipelines from the anesthesia side were gathered and align within the edge of table to avoid any entanglement with the rotating $\mathrm{C}$-arm (Figure 3). Then an initial scan was performed during end-inspiratory breath holding for needle pathway planning (Figure 4A). The needle entry point and angulation were visualized by projecting a laser-target cross onto the patient's surface (Figure 4B). We next introduced an 18-gauge marker needle into the patient's thorax during end-inspiratory breath-holding and corrected both needle orientation and positioning by projecting the planned, virtual needle trajectory onto the live fluoroscopic image. When the lesion was reached, the tumor was localized by placing a localization wire (DuaLok ${ }^{\circledR}$; Bard Peripheral Vascular, Inc., Tempe, AZ, USA). Superficial lesions were delineated by injecting PBV dye $(0.3-0.5 \mathrm{~mL}$, patent blue V $2.5 \%$; Guerbet). A post-procedural CBCT scan was 
obtained to confirm the accuracy of tumor localization.

\section{Data collection}

The demographic and clinical characteristics, anesthesia variable, tumor location, size and the minimum distance between tumor to pleura were collected retrospectively. The ratio of consolidation to total tumor size (CTR) were also calculated, according to the JCOG0201 definition (14). The details for localization, included localized position and method, total localization time, radiation dose, and operation findings and methods were also included.

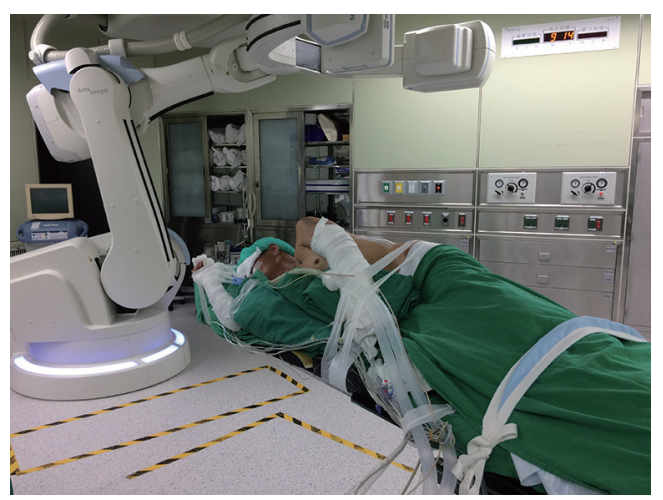

Figure 3 Patient positioning for iVATS. All pipelines were gathered and align within the edge of table to avoid any entanglement with the rotating C-arm.
The total localization was defined from the time the patient setting to planned position to CBCT back in place. Radiation dose was calculated as total skin dose (SD), which was collected from the "Exam Protocol" of the ARTIS zeego instrument. Descriptive statistics are summarized as medians [interquartile ranges (IQRs)] for continuous data and counts (percentages) for categorical variables. All analyses were performed with the Statistical Package for the Social Sciences (SPSS), version 20 (IBM, Armonk, NY, USA).

\section{Results}

From October $1^{\text {st }} 2016$ to July $31^{\text {st }} 2017,14$ patients with GGOs underwent iVATS. The general characteristic of the 14 patients are shown in Table 1. The median size for these patients is $7 \mathrm{~mm}$ (IQR: $4-10 \mathrm{~mm}$ ), whereas their median distance from the pleural surface was $8.5 \mathrm{~mm}$ (IQR: 0-11 mm). The median tumor depth-to-size (D-S) ratio was 1.16 (IQR: $0-2.3$ ). The median CTR was 0.42 (IQR: $0-0.57)$. There are 7 lesions located at right side and the other 7 located at left side.

The details of localization were summarized on the Table 2. Ten patients were placed in lateral decubitus position and the other 4 as supine or prone position, which need re-positioned for surgery after localization completed. All of the GGOs were visible on intraoperative CBCT images. Four (28.6\%) patients received localization with wire and the other 10 patients with dye.
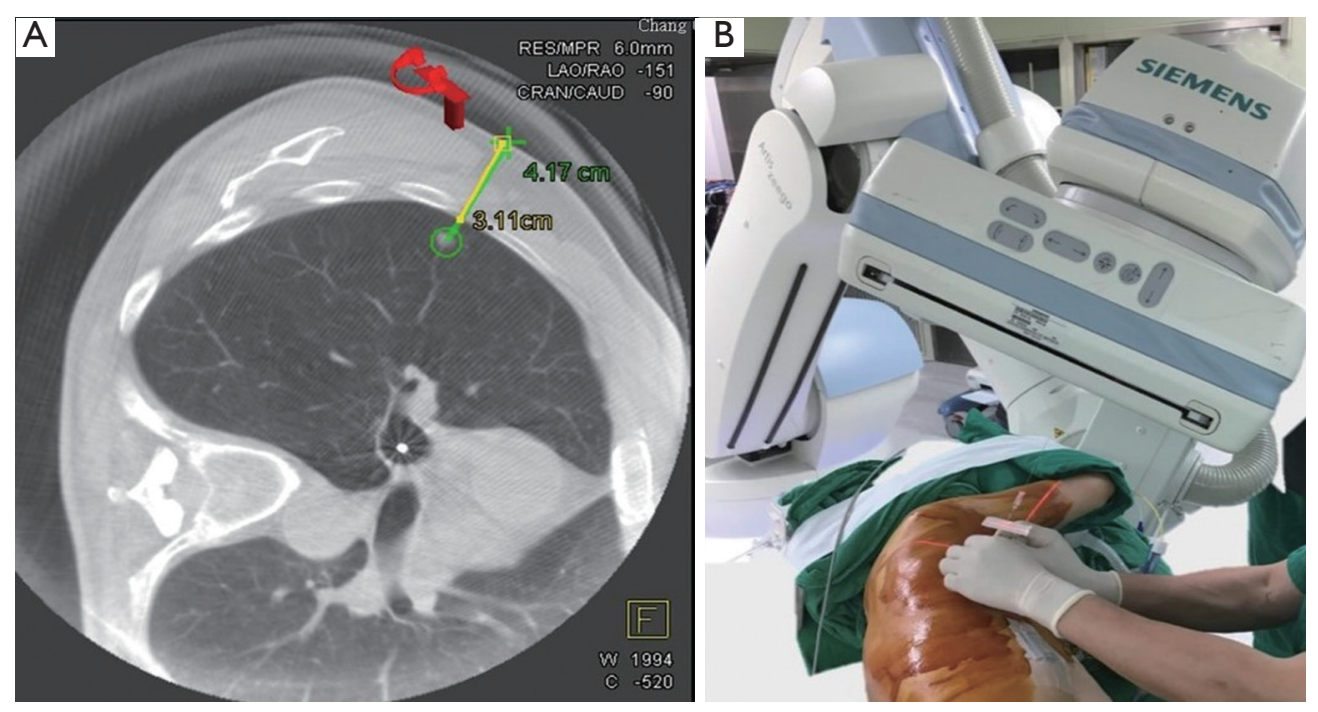

Figure 4 The process of needle puncture. (A) CBCT image for needle pathway planning; (B) the needle entry point and angulation were visualized by projecting a laser-target cross onto the patient's surface according to planned needle pathway. 
Table 1 General characteristic of the 14 patients

\begin{tabular}{|c|c|c|c|c|c|c|c|c|}
\hline No. & Age, years & Gender & ASA & Tumor location & Tumor size (mm) & Distance to pleura $(\mathrm{mm})$ & D-S ratio & CTR \\
\hline 1 & 21 & Male & 3 & LLL & 4 & 13 & 3.25 & 0.67 \\
\hline 2 & 63 & Male & 3 & RLL & 5 & 6 & 1.2 & 0.45 \\
\hline 3 & 44 & Male & 3 & LUL & 3 & 8 & 2.67 & 0.45 \\
\hline 4 & 37 & Male & 3 & LUL & 4 & 0 & 0 & 0 \\
\hline 5 & 49 & Female & 3 & RLL & 5 & 0 & 0 & 0 \\
\hline 6 & 18 & Male & 2 & LUL & 8 & 11 & 1.38 & 0.4 \\
\hline 7 & 60 & Male & 3 & RUL & 3.5 & 35 & 10 & 0.57 \\
\hline 8 & 50 & Female & 3 & RUL & 9 & 11 & 1.22 & 0 \\
\hline 9 & 72 & Female & 3 & RUL & 14 & 11 & 0.79 & 0.71 \\
\hline 10 & 66 & Male & 3 & $\mathrm{RML}$ & 11 & 7 & 0.64 & 0.82 \\
\hline 11 & 41 & Female & 3 & LLL & 6 & 14 & 2.33 & 0 \\
\hline 12 & 60 & Female & 3 & LUL & 8 & 9 & 1.13 & 0.57 \\
\hline 13 & 47 & Male & 3 & RUL & 10 & 0 & 0 & 0 \\
\hline 14 & 50 & Male & 3 & LUL & 10 & 0 & 0 & 0 \\
\hline Median & 49.5 & - & - & - & 7 & 8.5 & 1.16 & 0.42 \\
\hline IQR & $39-63$ & - & - & - & $4-10$ & $0-11$ & $0-2.33$ & $0-0.57$ \\
\hline
\end{tabular}

ASA, American Society of Anesthesiologists; D-S ratio, depth-to-size ratio; RUL, right upper lobe; RML, right middle lobe; RLL, right lower lobe; LUL, left upper lobe; LLL, left lower lobe; IQR, interquartile range; CTR, consolidation-to-tumor ratio.

The median of SD radiation dose was $106.3 \mathrm{mGy}$ (IQR: 83.3-198.5 mGy). All of the lesions were visible on intraoperative CBCT images and localizations were successful in all patients with a median localization time of $22 \mathrm{~min}$ (IQR:16-44 min). There was no post-localization complication such as pneumothorax, hemothorax, etc.

All of the lesions were localized successfully and were removed by sublobar resection. The final pathological reports showed 6 primary lung cancers, 2 metastases, and 6 benign lesions. In presence of an intraoperative frozensection diagnosis of malignancies or precancerous lesions, an additional systematic lymph node dissection was performed. The median hospital stay after operation was 4 days with no in hospital mortality.

\section{Discussion}

To our knowledge, this is the largest study to report the use iVATS for localization and resection for GGOs. Our data indicated the high successful lesion targeting rate $(100 \%)$ of CBCT for localization of GGO lesion and acceptable procedure time (median: $22 \mathrm{~min}$ ), which was compatible to previous published result obtained in interventional CT-suite $(4,5,7)$. More importantly, by taking advantage of the hybrid OR, iVATS allows surgery to be carried out in a more timely fashion following lesion localization than conventional two-stages, which theoretically reduces the risk of wire dislodgement/dye fading and patient discomfort. Taken together, our findings suggest that iVATS offers a patient-centered surgical approach and may serve as a standard approach for treating GGOs.

Despite all the advantaged, there are also some challenges we faced during the development of iVATS. First, it has been reported that CBCT has relative low resolution compared to traditional multiple detector computed tomography (MDCT), and thus the detection of GGOs would be problematic (15). However, in our study, all lesions could be seen on pre-procedural CBCT image and the smallest one was $3 \mathrm{~mm}$. Second, the occurrence of collision between rotating $\mathrm{C}$-arm and table and gas pipelines. The acquisition of a Dyna-CT image requires a complete 200-degree rotation of the C-arm around the surgical table. Care must be taken to align all pipelines within the edge of table to avoid entanglement 
Table 2 Details of localization of the 14 patients

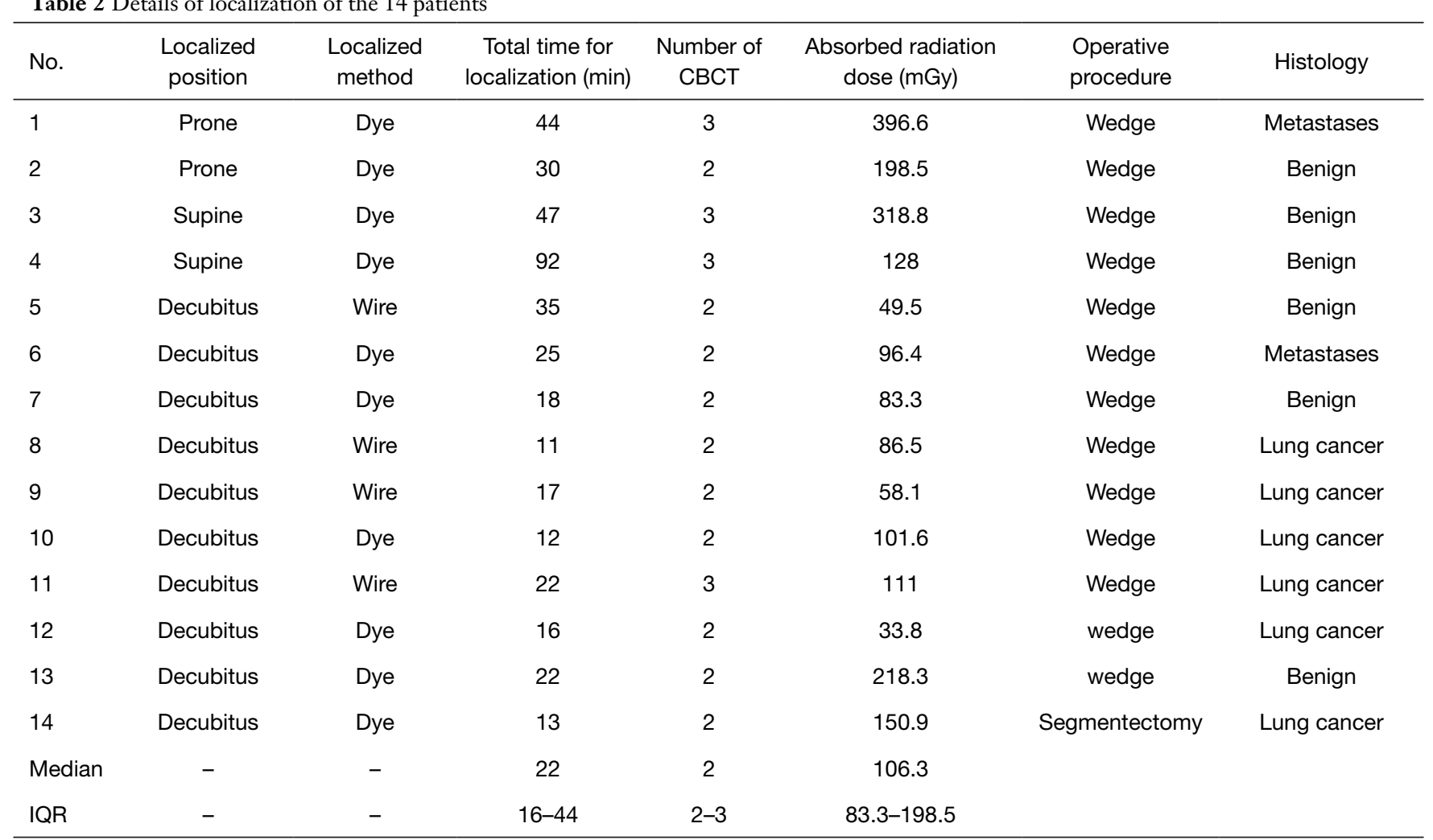

СВCT, C-arm cone-beam computed tomography.

\section{(Figure 3).}

Meanwhile, the field of visualization (FOV) is generally smaller than MDCT. In order to include both the target lung lesion and the needle entry site into a unique CBCT FOV, the surgical table might be sporadically placed too low, ultimately leading to a collision with the $\mathrm{C}$-arm. This concrete risk was especially evident when patients with a peripheral lung mass and/or a large chest cavity were placed in the lateral decubitus position. In our first four patients (\#1-4), we have to reposition patient from decubitus to either prone/supine position due to the occurrence of collision, which resulted in longer procedure time (44, 30, 47, $92 \mathrm{~min})$ and higher radiation exposure (396.6, 198.5, 318.8, $128 \mathrm{mGy}$ ). To circumvent this issue, we believed a better understanding of the limitation of reciprocal positioning of the patient, surgical table, and $\mathrm{C}$-arm is of paramount importance. We have identified several useful maneuvers (including table height management and adjustments of the patient position) that may be helpful to reduce the procedural time (16). The implementation of these maneuvers as we overcame the initial procedure-related learning curve resulted in an increase of successful GGOs localization in the lateral decubitus position (100\%) over the latter 10 patients.

Our findings need to be interpreted in the context of several limitations. First, the study cohort consisted of selected patients who had a single GGO requiring localization. The question as to whether iVATS can be safely applied to patients harboring two or more GGOs in need of localization remains open. Second, as the radiation dynamics of CBCT and MDCT are different, we could not estimate the real radiation exposure in iVATS patients from scanner-estimated dose. Future research is necessary to compare this novel technique with previously established localization methods in terms of diagnostic yields, complication rates, and costeffectiveness.

\section{Conclusions}

In conclusion, our result suggests the iVATS could be a helpful tool for single-stage localization and removal of GGOs. 


\section{Acknowledgements}

Funding: This study was financially supported by a grant (CMRPG3F1811) from the Chang Gung Memorial Hospital, Taiwan.

\section{Footnote}

Conflicts of Interest: The authors have no conflicts of interest to declare.

Ethical Statement: The study protocol was approved by the Institutional Review Board of the Chang Gung Memorial Hospital (CGMH-IRB 201600671A3). Written informed consent was obtained from all participants.

\section{References}

1. National Lung Screening Trial Research Team, Aberle DR, Adams AM, et al. Reduced lung-cancer mortality with low-dose computed tomographic screening. $\mathrm{N}$ Engl J Med 2011;365:395-409.

2. Gould MK, Donington J, Lynch WR, et al. Evaluation of individuals with pulmonary nodules: when is it lung cancer? Diagnosis and management of lung cancer, $3 \mathrm{rd}$ ed: American College of Chest Physicians evidence-based clinical practice guidelines. Chest 2013;143:e93S-120S.

3. Suzuki K, Nagai K, Yoshida J, et al. Video-assisted thoracoscopic surgery for small indeterminate pulmonary nodules: indications for preoperative marking. Chest 1999;115:563-8.

4. Kim GR, Hur J, Lee SM, et al. CT fluoroscopy-guided lung biopsy versus conventional CT-guided lung biopsy: a prospective controlled study to assess radiation doses and diagnostic performance. Eur Radiol 2011;21:232-9.

5. Naidich DP, Bankier AA, MacMahon H, et al. Recommendations for the management of subsolid pulmonary nodules detected at CT: a statement from the Fleischner Society. Radiology 2013;266:304-17.

6. Tomiyama N, Yasuhara Y, Nakajima Y, et al. CTguided needle biopsy of lung lesions: a survey of severe

doi: 10.21037 /jovs.2017.09.08

Cite this article as: Fang HY, Chao YK, Hsieh MJ, Wen CT, Ho PH, Tang WJ, Liu YH. Image-guided video-assisted thoracoscopic surgery for small ground glass opacities: a case series. J Vis Surg 2017;3:142. complication based on 9783 biopsies in Japan. Eur J Radiol 2006;59:60-4.

7. Ichinose J, Kohno T, Fujimori S, et al. Efficacy and complications of computed tomography-guided hook wire localization. Ann Thorac Surg 2013;96:1203-8.

8. Kostrzewa M, Kara K, Rathmann N, et al. Computed Tomography-Assisted Thoracoscopic Surgery: A Novel, Innovative Approach in Patients With Deep Intrapulmonary Lesions of Unknown Malignant Status. Invest Radiol 2017;52:374-80.

9. Yan GW, Bhetuwal A, Yan GW, et al. A Systematic Review and Meta-Analysis of C-Arm Cone-Beam CT-Guided Percutaneous Transthoracic Needle Biopsy of Lung Nodules. Pol J Radiol 2017;82:152-60.

10. Yang SM, Ko WC, Lin MW, et al. Image-guided thoracoscopic surgery with dye localization in a hybrid operating room. J Thorac Dis 2016;8:S681-9.

11. Hansell DM, Bankier AA, MacMahon H, et al. Fleischner Society: glossary of terms for thoracic imaging. Radiology 2008;246:697-722.

12. Hsieh CP, Hsieh MJ, Fang HY, et al. Imaging-guided thoracoscopic resection of a ground-glass opacity lesion in a hybrid operating room equipped with a robotic C-arm CT system. J Thorac Dis 2017;9:E416-9.

13. Fang HY, Chao YK, Hsieh MJ, et al. Image-guided VATS (iVATS) workflow. Asvide 2017;4:449. Available online: http://www.asvide.com/articles/1752

14. Suzuki K, Koike T, Asakawa T, et al. A prospective radiological study of thin-section computed tomography to predict pathological noninvasiveness in peripheral clinical IA lung cancer (Japan Clinical Oncology Group 0201). J Thorac Oncol 2011;6:751-6.

15. Rouzé S, de Latour B, Flécher E, et al. Small pulmonary nodule localization with cone beam computed tomography during video-assisted thoracic surgery: a feasibility study. Interact Cardiovasc Thorac Surg 2016;22:705-11.

16. Hsieh MJ, Fang HY, Lin CC, et al. Single-stage localization and removal of small lung nodules through image-guided video-assisted thoracoscopic surgery. Eur J Cardiothorac Surg 2017. [Epub ahead of print]. 